\title{
Subacute Facial Nerve Paralysis Following Cyberknife Treatment for Vestibular Schwannoma
}

\author{
Peng $\mathrm{KA}^{1}$, Chen $\mathrm{Y}^{\mathrm{R}} \mathrm{R}^{2}$, Chen $\mathrm{D}^{3}$, Chen $\mathrm{BS}^{1}$, Johnson $\mathrm{E}^{\star 2}$ and Brackmann $\mathrm{DE}^{1}$ \\ ${ }^{1}$ House Clinic, Los Angeles, California, USA \\ ${ }^{2}$ Department of Neurosurgery, Stanford Healthcare, Palo Alto, California, USA \\ ${ }^{3}$ Department of Medicine, Stanford Healthcare, Palo Alto, California, USA
}

*Corresponding author: Johnson E, BS, Stanford Healthcare, 300 Pasteur Drive, Stanford, CA 94305, USA, Fax: (650) 725-0390, Tel: (347) 241-6147, E-mail: ej586@stanford.edu

Citation: Peng KA, Chen Y-R, Chen D, Chen BS, Johnson E, et al. (2018) Subacute Facial Nerve Paralysis Following Cyberknife Treatment for Vestibular Schwannoma. J Cas Rep Stud 6(1): 103. doi: 10.15744/23489820.6.103

Received Date: January 28, 2018 Accepted Date: February 26, 2018 Published Date: February 28, 2018

\begin{abstract}
Facial nerve paralysis may occur along the natural progression of an untreated vestibular schwannoma, as a surgical complication following vestibular schwannoma resection, or as a late sequela of stereotactic radiation. However, facial paralysis occurring as a subacute complication of CyberKnife (Accuray, Inc., Sunnyvale, CA, USA) stereotactic radiation has not previously been reported. A 47-year-old male presented with a right vestibular schwannoma measuring $3.1 \mathrm{~cm}$ in greatest dimension with House-Brackmann grade I/VI facial function and American Academy of Otolaryngology - Head and Neck Surgery class a hearing. After deferring surgery, he elected to undergo CyberKnife stereotactic radiation to a total dose of $18 \mathrm{~Gy}$ in three stages. Within three months, the patient noticed gradual ipsilateral facial paralysis that progressed to House-Brackmann grade VI/VI over the course of four weeks. The patient's facial function was assessed continuously for one year with no volitional function or resting tone. He thus underwent a hypoglossal-facial transfer, restoring facial function to House-Brackmann IV/VI, with durable results at a follow-up length of five years. To our knowledge, this is the first reported case of subacute facial paralysis following CyberKnife stereotactic radiation for vestibular schwannoma.
\end{abstract}

Keywords: Facial Nerve Paralysis; Cyberknife Treatment; Vestibular Schwannoma

List of abbreviations: MRI: Magnetic Resonance Imaging; IAC: Internal Auditory Canal; WRS: Word Recognition Score

\section{Introduction}

Facial nerve paralysis may occur in the setting of vestibular schwannoma as a sequela of the natural history of an untreated tumor, as a complication of surgery, or as a late complication of stereotactic radiation. Estimates of facial nerve preservation following surgical resection, corresponding to grades I or II on the House-Brackmann scale [1], have been estimated at roughly $60 \%$ immediately postoperatively to $80-90 \%$ at long-term follow-up $[2,3]$. On the other hand, stereotactic radiation has been associated with an overall facial nerve preservation rate of $96.2 \%$ at long-term follow-up [4]. In particular, CyberKnife (Accuray, Inc., Sunnyvale, CA, USA) has been associated with excellent facial nerve preservation [5], with one series noting $100 \%$ preservation of facial function in 60 patients [6].

While facial nerve neuropathy in the setting of long-term outcomes following CyberKnife radiosurgery has been documented [7], facial nerve paralysis has not previously been reported as a subacute complication of stereotactic radiation for vestibular schwannoma. Here, we report a case of complete facial paralysis occurring within 3 months following CyberKnife stereotactic radiation for vestibular schwannoma.

\section{Case Presentation}

Informed verbal consent was obtained by the patient reported in this study.

A 47-year-old male presented to the neurology clinic with a chief complaint of episodic vertigo. The patient had no hearing complaints, and facial nerve function was noted to be I/VI. Magnetic resonance imaging (MRI) of the internal auditory canals (IAC) was performed, revealing a right-sided vestibular schwannoma measuring 3.1 by 2.7 by $2.4 \mathrm{~cm}$, with extension into the IAC (Figure 1A). Subsequently, the patient was referred to the neurotologic clinic, where an audiogram revealed a right-sided mild high- 
frequency hearing loss with a word recognition score (WRS) of 100\%, corresponding to American Academy of Otolaryngology - Head and Neck Surgery hearing class A [8].

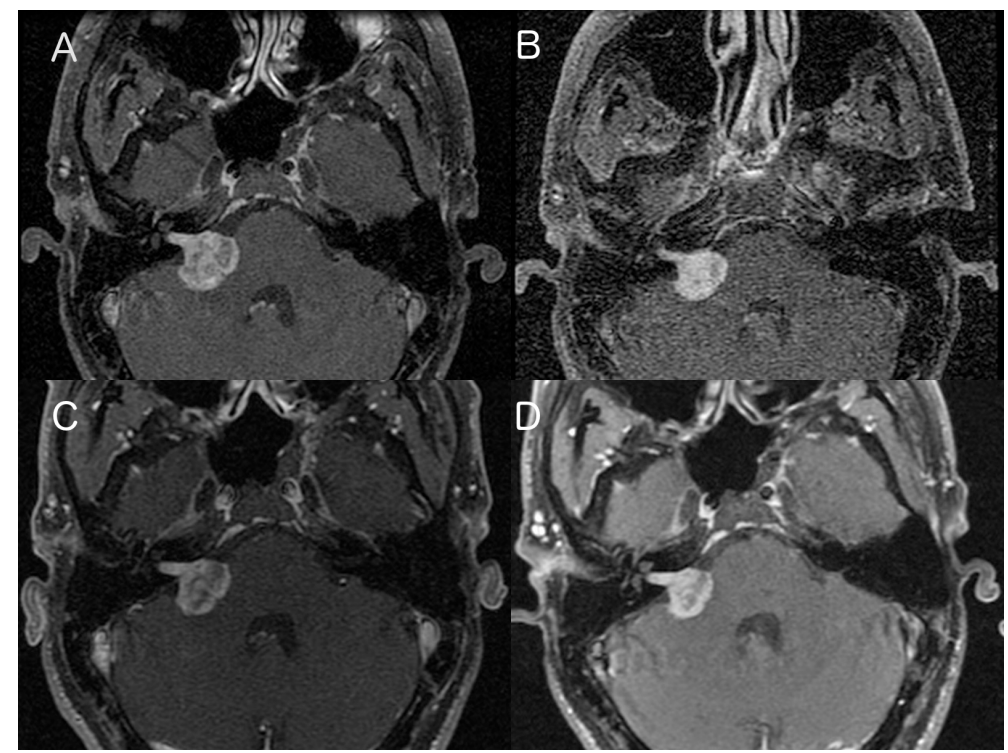

Figure 1: Horizontal MRI sections, showing Vestibular Schwannoma with extension into IAC (A) Pretreatment; (B) 6 weeks; (C) 12 weeks; (D) 30 months

Following a discussion of management options, including surgery, stereotactic radiation, and observation, the patient deferred surgery and elected to proceed with stereotactic radiation. He was referred to the neurosurgical clinic for CyberKnife evaluation, and was deemed an acceptable candidate.

Over the course of three days, the patient underwent CyberKnife stereotactic radiation to his right vestibular schwannoma with a dose of 18 Gy delivered to the $78 \%$ isodose line using a $15 \mathrm{~mm}$ collimator. The maximal dose within the center of the treatment volume was $23.1 \mathrm{~Gy}$, and the volume treated was $10.4 \mathrm{~cm}^{3}$ (Figure 2). Dexamethasone $8 \mathrm{mg}$ was administered orally after each of the three treatment sessions.

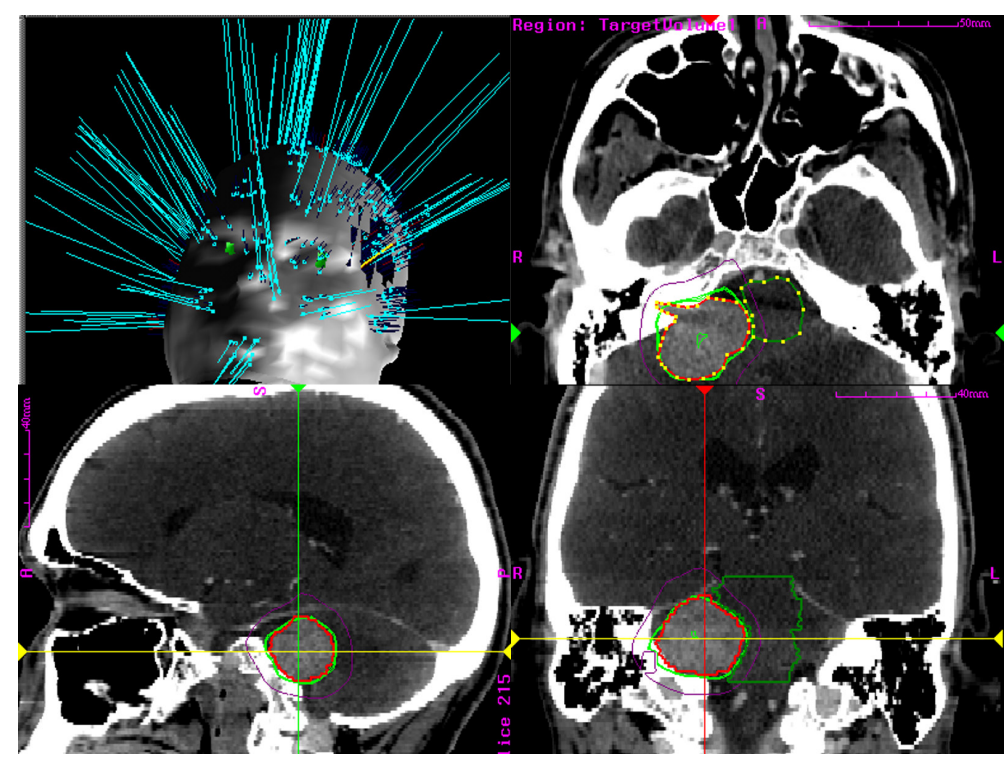

Figure 2: MRI 6 weeks after CyberKnife stereotactic radiation

The patient had no immediate complications, and MRI performed 6 weeks following stereotactic radiation revealed no changes in the treated lesion (Figure 1B). However, approximately 12 weeks following radiation, he began to notice facial asymmetry. He then noticed progressive headache and right-sided facial weakness over the course of four weeks, after which he was noted to have complete paralysis, House-Brackmann grade VI. MRI was repeated, and revealed central tumor necrosis and enlargement of the tumor to $3.4 \mathrm{~cm}$ (Figure 1C). The patient was started on a course of oral dexamethasone daily.

Serial imaging performed thereafter revealed a peak tumor size of $3.8 \mathrm{~cm}$ in greatest dimension. The patient developed transient papilledema and radiological evidence of hydrocephalus, which were managed medically. Eight months following stereotactic radiation, papilledema and hydrocephalus had both resolved, and oral dexamethasone was discontinued after a taper. However, facial function remained at House-Brackmann grade VI. 
One year following the onset of facial paralysis, the patient presented again to the neurotologic clinic. He endorsed no serviceable hearing on the affected side, and audiological testing confirmed a WRS of $0 \%$ accompanying a mild sloping to profound sensorineural loss. Examination confirmed persistent House-Brackmann grade VI paralysis, and the patient elected to undergo hypoglossal-facial anastomosis for dynamic reanimation of his paralyzed face. By 18 months following surgery, the patient had restoration of House-Brackmann grade IV facial function, which remained stable at the last follow-up of 5 years postoperatively. Repeat MRI performed approximately two and a half years following stereotactic radiation revealed no progression in tumor size (Figure 1D).

\section{Discussion}

Facial nerve paralysis is recognized as a potential complication of surgical management or a sequela of stereotactic radiation that carries significant implications for quality of life following treatment [9]. Rates of anatomic preservation of the facial nerve following microsurgery range from $93-97 \%$ in large series $[2,10]$. Normal or near-normal facial function following surgery, defined as grade I or II on the House-Brackmann scale, is achieved in close to $90 \%$ of patients [2].

Delayed facial palsy, defined as deterioration of facial nerve function more than three days following treatment, has been reported as an uncommon sequela of microsurgery for vestibular schwannoma. Rates of delayed facial palsy range from 5 to $26 \%$, but the prognosis is excellent, with a vast majority of patients noting full recovery to immediate postoperative function within one year $[11,12]$. Potential etiologies for delayed facial palsy include reactivation of herpes simplex virus and varicella zoster virus [13], and because of this, routine antiviral therapy following vestibular schwannoma resection has been advocated [14].

Facial nerve dysfunction following stereotactic radiation is relatively uncommon, with reported rates ranging from $0 \%$ to $7 \%$ $[6,15,16]$. Facial nerve dysfunction in these cases is hypothesized to result from compromised microvasculature in addition to progressive inflammation and fibrosis, a theory corroborated by intraoperative findings reported at the time of surgical salvage [17]. Overall, however, facial nerve paralysis following stereotactic radiation, including the CyberKnife system, is uncommon if facial nerve function is intact prior to treatment $[4,7,18-20]$.

In the present case, a patient with a $3.1 \mathrm{~cm}$ vestibular schwannoma presenting with House-Brackmann grade I facial function experienced progressive, ultimately complete facial paralysis beginning fewer than three months following stereotactic radiation with CyberKnife. To our knowledge, this is the first such report in the literature. After observation for one year, the patient was noted to have no facial function, remaining at House-Brackmann grade VI. Function was restored to House-Brackmann grade IV following a hypoglossal-facial anastomosis.

Various authors have suggested that cranial nerve dysfunction, including facial paralysis, following stereotactic radiosurgery correlates strongly with minimum radiation dose as well as total radiation dose [21]. Hypofractionation has additionally been associated with a lower incidence of cranial nerve dysfunction following stereotactic radiation [22]. Although hypofractionation was indeed employed in the present case, a significant factor predisposing to a poorer outcome following stereotactic radiation was the size of the patient's tumor $(3.1 \mathrm{~cm})$.

\section{Conclusion}

To our knowledge, this is the first report of subacute-onset facial nerve paralysis following CyberKnife stereotactic radiation. Facial paralysis, whether subacute or delayed, remains a known complication of stereotactic radiation for vestibular schwannoma. The possibility of such - as embodied by the present case-carries implications for patient counseling when determining the optimal management of a vestibular schwannoma.

\section{References}

1. House JW, Brackmann DE (1985) Facial nerve grading system. Otolaryngol Head Neck Surg 93: 146-7.

2. Sampath P, Holliday MJ, Brem H, Niparko JK, Long DM (1997) Facial nerve injury in acoustic neuroma (vestibular schwannoma) surgery: etiology and prevention. J Neurosurg 87: 60-6.

3. Brackmann DE, Cullen RD, Fisher LM (2007) Facial nerve function after translabyrinthine vestibular schwannoma surgery. Otolaryngol Head Neck Surg 136: 773-7.

4. Yang I, Sughrue ME, Han SJ, Fang S, Aranda D, et al. (2009) Facial nerve preservation after vestibular schwannoma Gamma Knife radiosurgery. J Neurooncol 93: 41-8.

5. Teo M, Zhang M, Li A, Thompson PA, Tayag AT, et al. (2016) The outcome of hypofractionated sterotatic radiosurgery for large vestibular schwannomas. World Neurosurg 93: 398-409.

6. Sakamoto GT, Blevins N, Gibbs IC (2009) Cyberknife radiotherapy for vestibular schwannoma. Otolaryngol Clin North Am 42: 665-75.

7. Mahboubi H, Sahyouni R, Moshtaghi O, Tadokoro K, Ghavami Y, et al. (2017) CyberKnife for treatment of vestibular schwannoma: a meta-analysis. Otolaryngol Head Neck Surg 157: 7-15.

8. Board of Directors of the American Academy of Otolaryngology-Head and Neck Surgery (1995) Committee on Hearing and Equilibrium guidelines for the evaluation of hearing preservation in acoustic neuroma (vestibular schwannoma). American Academy of Otolaryngology-Head and Neck Surgery Foundation, INC. Otolaryngol Head Neck Surg 113: 179-80. 
9. Lassaletta L, Alfonso C, Del Rio L, Roda JM, Gavilan J (2006) Impact of facial dysfunction on quality of life after vestibular schwannoma surgery. Ann Otol Rhinol Laryngol 115: 694-8.

10. Samii M, Matthies C (1997) Management of 1000 vestibular schwannomas (acoustic neuromas): the facial nerve--preservation and restitution of function. Neurosurgery 40: 684-95.

11. Grant GA, Rostomily RR, Kim DK, Mayberg MR, Farrell D, et al. (2002) Delayed facial palsy after resection of vestibular schwannoma. J Neurosurg 97: 93-6. 12. Magliulo G, D'Amico R, Di Cello P (2003) Delayed facial palsy after vestibular schwannoma resection: clinical data and prognosis. J Otolaryngol 32: 400-4.

13. Franco-Vidal V, Nguyen DQ, Guerin J, Darrouzet V (2004) Delayed facial paralysis after vestibular schwannoma surgery: role of herpes viruses reactivation-our experience in eight cases. Otol Neurotol 25: 805-10.

14. Brackmann DE, Fisher LM, Hansen M, Halim A, Slattery WH, et al. (2008) The effect of famciclovir on delayed facial paralysis after acoustic tumor resection. Laryngoscope. 118: 1617-20.

15. Meijer OW, Vandertop WP, Baayen JC, Slotman BJ (2003) Single-fraction vs. fractionated linac-based stereotactic radiosurgery for vestibular schwannoma: a single-institution study. Int J Radiat Oncol Biol Phys 56: 1390-6.

16. Chung WY, Liu KD, Shiau CY, Wu HM, Wang LW, et al. (2013) Gamma knife surgery for vestibular schwannoma: 10-year experience of 195 cases. J Neurosurg 119: 87-97.

17. Friedman RA, Brackmann DE, Hitselberger WE, Schwartz MS, Iqbal Z, et al. (2005) Surgical salvage after failed irradiation for vestibular schwannoma. Laryngoscope 115: 1827-32.

18. Ishihara H, Saito K, Nishizaki T, Kajiwara K, Nomura S, et al. (2004) CyberKnife radiosurgery for vestibular schwannoma. Minim Invasive Neurosurg 47: 290-3. 19. Hempel JM, Hempel E, Wowra B, Schichor Ch, Muacevic A, et al. (2006) Functional outcome after gamma knife treatment in vestibular schwannoma. Eur Arch Otorhinolaryngol 263: 714-8.

20. Jumeau R, Bondiau PY, Parietti-Winkler C, Salleron J, Civit T, et al. (2016) Vestibular schwannomas treated with CyberKnife: clinical outcomes. Tumori 102: 569-73.

21. Flickinger JC, Kondziolka D, Lunsford LD (1996) Dose and diameter relationships for facial, trigeminal, and acoustic neuropathies following acoustic neuroma radiosurgery. Radiother Oncol 41: 215-9.

22. Ju DT, Lin JW, Lin MS, Lee LM, Tseng HM, et al. (2008) Hypofractionated CyberKnife stereotactic radiosurgery for acoustic neuromas with and without association to neurofibromatosis Type 2. Acta Neurochir Suppl. 101: 169-73.

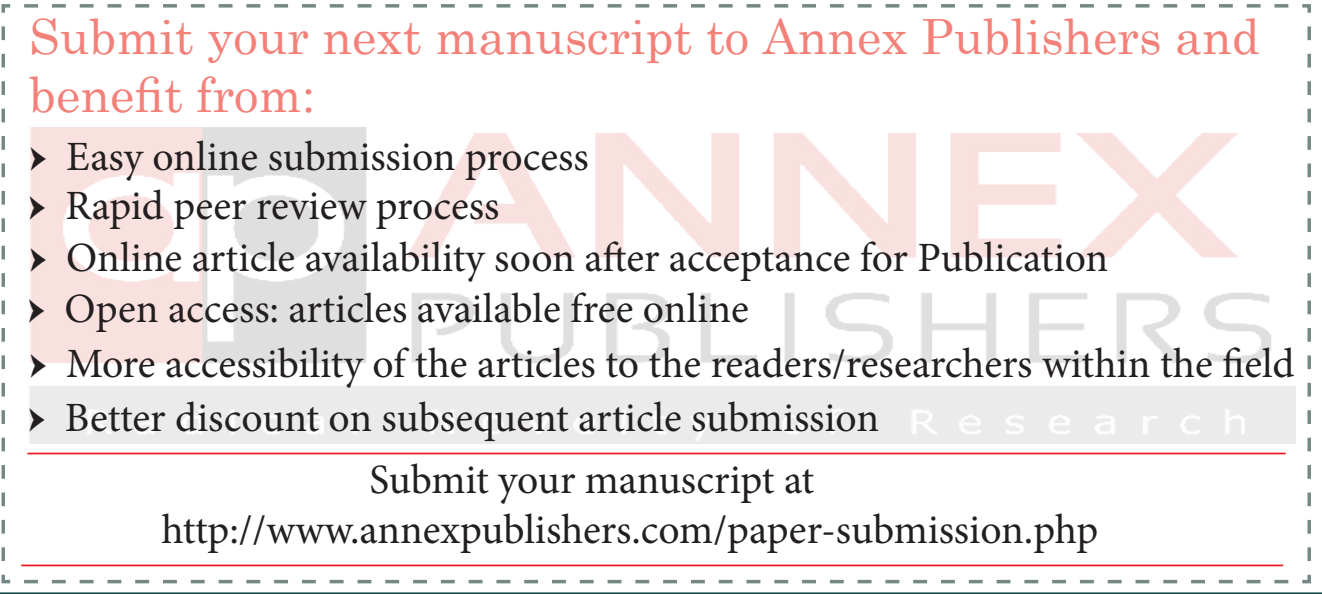

